\title{
Corrigendum
}

\section{Corrigendum to "Study on the Gap Flow Simulation in EDM Small Hole Machining with Ti Alloy"}

\author{
Shengfang Zhang, Wenchao Zhang, Yu Liu, Fujian Ma, Chong Su, and Zhihua Sha \\ School of Mechanical Engineering, Dalian Jiaotong University, Dalian 116028, China \\ Correspondence should be addressed to Yu Liu; liuyu_ly12@126.com \\ Received 25 September 2017; Accepted 12 October 2017; Published 24 October 2017 \\ Copyright (C) 2017 Shengfang Zhang et al. This is an open access article distributed under the Creative Commons Attribution \\ License, which permits unrestricted use, distribution, and reproduction in any medium, provided the original work is properly \\ cited.
}

In the article titled "Study on the Gap Flow Simulation in EDM Small Hole Machining with Ti Alloy" [1], Dr. Wenchao Zhang was incorrectly listed as the corresponding author. The corresponding author is Dr. Yu Liu.

\section{References}

[1] S. Zhang, W. Zhang, Y. Liu, F. Ma, C. Su, and Z. Sha, "Study on the gap flow simulation in EDM small hole machining with $\mathrm{Ti}$ alloy," Advances in Materials Science and Engineering, vol. 2017, Article ID 8408793, 23 pages, 2017. 

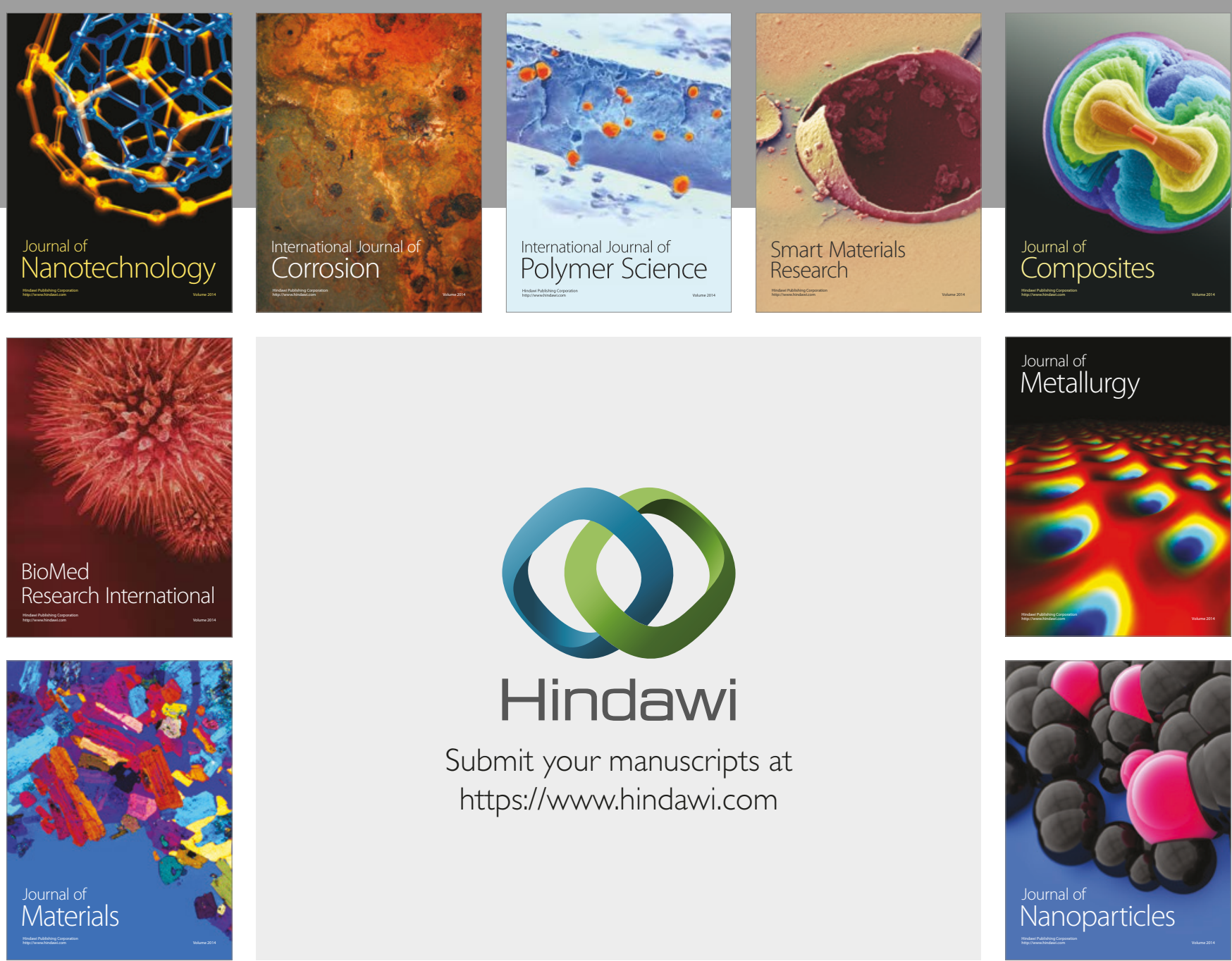

\section{Hindawi}

Submit your manuscripts at

https://www.hindawi.com
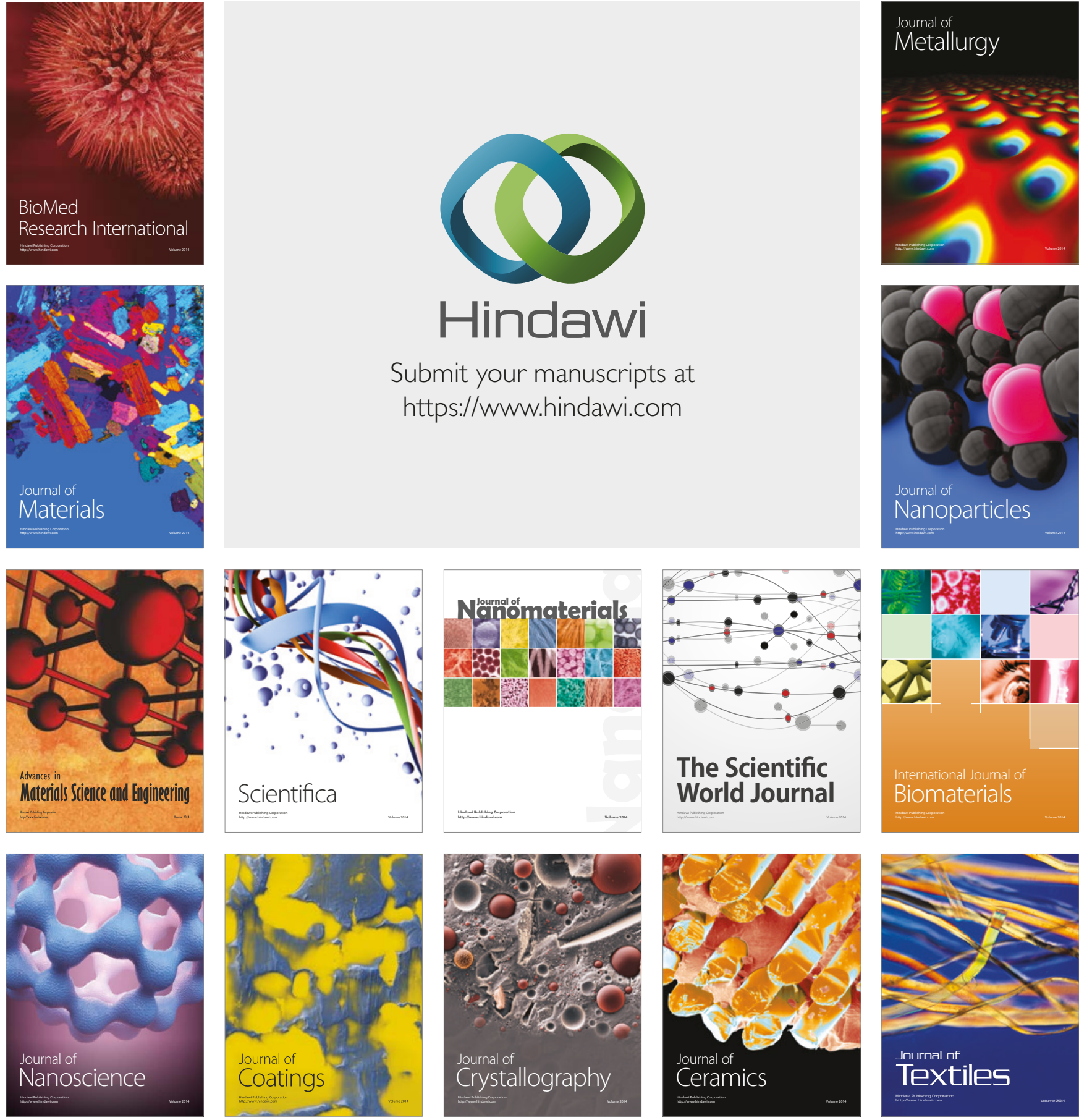

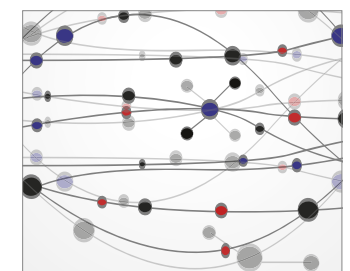

The Scientific World Journal
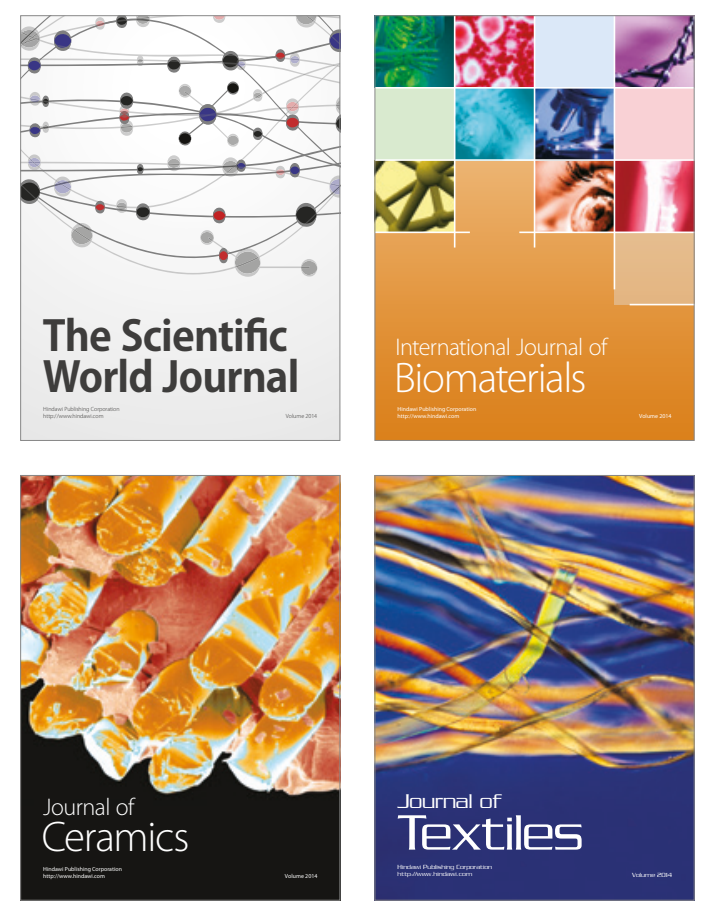